\title{
INFLUÊNCIA DA PROFUNDIDADE DE SEMEADURA E DA COMPACTAÇÃO DO SOLO SOBRE A SEMENTE NA PRODUTIVIDADE DO AMENDOIM
}

\author{
Influence of sowing depth and the compacting of the ground on the seed in \\ the peanut productivity \\ Danilo Cesar Checchio Grotta ${ }^{1}$, Carlos Eduardo Angeli Furlani ${ }^{2}$, Rouverson Pereira Silva ${ }^{2}$, \\ Gustavo Naves dos Reis ${ }^{1}$, Jorge Wilson Cortez ${ }^{1}$, Pamela José Alves ${ }^{3}$
}

\begin{abstract}
RESUMO
A profundidade de semeadura e a compactação do solo sobre a semente são específicas para cada cultura e dependem de vários atributos. Assim, objetivou-se avaliar a influência da compactação do solo sobre a semente e de diferentes profundidades de semeadura em um LATOSSOLO VERMELHO Eutroférrico típico na emergência e produtividade do amendoim. As cargas verticais impostas para compactação foram $0 ; 98,1 ; 196,2$ e 294,3 $\mathrm{N}$ e as profundidades de semeadura de 0,04, 0,06 e 0,08 m. A compactação do solo na linha de semeadura não interferiu na produtividade da cultura. As profundidades de 0,04 e $0,06 \mathrm{~m}$ foram mais favoráveis para o desenvolvimento da cultura do amendoim. A interação profundidade versus compactação não apresentou correlação.
\end{abstract}

Termos para indexação: Amendoim, dias para emergência, massa de grãos.

\section{ABSTRACT}

The sowing depth and compaction of the ground on seed are specific for each culture and depend on some attributes. Thus, are aimed to evaluate the influence of the compaction of ground on the seed in different sowing depths in a typical eutrustox red latosoil in peanut emergency and productivity. The vertical loads on the imposed for compacting were $0 ; 98.1 ; 196.2$ and $294.3 \mathrm{~N}$ and sowing depths of $0.04,0.06$ and $0.08 \mathrm{~m}$. The compaction of the ground in the sowing line did not interfere in the development of the culture. The depths of 0.04 and $0.06 \mathrm{~m}$ were more favorable for the growing of the culture of peanut. The interaction productivity versus compacting did not show correlation.

Index terms: Peanut, days for emergency, mass of grains.

(Recebido em 21 de julho de 2006 e aprovado em 19 de abril de 2007)

\section{INTRODUÇÃO}

O amendoim é considerado uma das mais importantes leguminosas, não só por sua expressão econômica, mas também por ser rico em proteínas (48\%) e com valor energético de reconhecida qualidade. O amendoim é muito recomendado em programas de rotação de culturas, por ser de ciclo curto, relativamente resistente à seca e totalmente mecanizado.

Em São Paulo, as principais regiões produtoras são Ribeirão Preto e Marília, na reforma de canaviais e pastagens, respectivamente. $\mathrm{O}$ aumento da produção de amendoim no País é de grande importância, pois além de atender às necessidades internas, o Brasil tem boas perspectivas de aumentar sua participação no mercado externo.

As rodas compactadoras das semeadoras têm por função, em algumas máquinas, exercer o controle de profundidade e firmar o solo em torno e/ou sobre a semente. Entretanto, a influência de diversos modelos de rodas compactadoras e sua pressão sobre o solo no desenvolvimento das culturas implantadas, são fatores pouco conhecidos (SILVA, 2002).

Para se obter êxito na exploração de uma cultura, além da adoção de uma série de cuidados indispensáveis, é fundamental conseguir, de imediato, uma boa porcentagem de emergência das plântulas, que propiciará uma população adequada ao melhor desenvolvimento da cultura. Tal emergência está diretamente relacionada à germinação. Segundo Ortolani et al. (1986), durante a germinação das sementes, três fatores são fundamentais e devem estar presentes adequadamente: calor, umidade e oxigênio. Para a emergência, um quarto fator torna-se importante, a profundidade.

As rodas de compactação têm a função de aumentar o contato das sementes com o solo para melhorar a absorção de água, garantindo melhor germinação (PACHECO, 1994).

Hummel et al. (1981) afirmam que a pressão aplicada sobre o solo pelas rodas compactadoras pode, ou não, ser

'Engenheiros Agrônomos, Doutorandos - Departamento de Engenharia Rural/DER - Universidade Estadual Paulista/UNESP - Via de Acesso Professor Paulo Donato Castellane, s/n-14884-900 - Jaboticabal, SP-dcgrotta@zipmail.com.br; gn_reis@yahoo.com.br; jorge.cortez@posgrand.fcav.unesp.br-Bolsista CNPq 2Doutores, Professores Adjunto - Departamento de Engenharia Rural/DER - Universidade Estadual Paulista/UNESP - Via de Acesso Professor Paulo Donato Castellane, s/n - 14884-900 - Jaboticabal, SP - furlani@fcav.unesp.br; rouverson@fcav.unesp.br - Bolsista CNPq

${ }^{3}$ Zootecnista, Mestranda - Departamento de Engenharia Rural/DER - Universidade Estadual Paulista/UNESP - Via de Acesso Professor Paulo Donato Castellane, s/n - 14884-900 - Jaboticabal, SP - pjalves@hotmail.com 
benéfica para a semente, dependendo do nível de pressão, do desenho da roda, do teor de água do solo e das condições climáticas durante o período de semeadura e emergência.

Savi Filho (1980), estudando técnicas adequadas para o cultivo do amendoim, recomenda a semeadura dessa

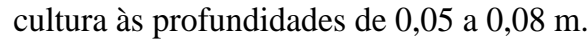

Furlani et al. (2001) combinando três profundidades de semeadura da cultura do milho com quatro níveis de compactação do solo sobre a semente, não encontraram influência desses fatores sobre o número médio de dias para emergência das plântulas.

Segundo Silva (2002) a profundidade de semeadura foi o fator que mais influenciou a emergência e o desenvolvimento vegetativo da cultura no milho.

Pressupõe-se que o aumento da profundidade de semeadura e da compactação sobre o solo poderá aumentar o número de dias para germinação e interferir nos demais parâmetros de desenvolvimento da cultura do amendoim e, nesse contexto, o presente trabalho visou avaliar a profundidade de semeadura e a compactação sobre o solo, na emergência e produtividade do amendoim.

\section{MATERIAL E MÉTODOS}

O trabalho foi realizado na área experimental do Laboratório de Máquinas e Mecanização Agrícola (LAMMA) do Departamento de Engenharia Rural da Faculdade de Ciências Agrárias e Veterinárias da UNESP, Campus de Jaboticabal/SP, localizado nas coordenadas geodésicas: $21^{\circ} 15^{\prime}$ Latitude Sul e $48^{\circ} 18^{\prime}$ Longitude Oeste, com altitude média de $570 \mathrm{~m}$ e clima Cwa (subtropical), de acordo com a classificação de Köeppen.

O solo da área experimental é classificado por Andrioli \& Centurion (1999) como LATOSSOLO VERMELHO Eutroférrico típico, textura muito argilosa, A moderado, caulinítico-oxálico, relevo suave ondulado.

O delineamento estatístico consistiu de blocos ao acaso, em esquema fatorial de 4 x 3 , com três repetições, sendo utilizadas quatro intensidades de compactação do solo sobre a semente e três profundidades de semeadura.

A área caracteriza-se por duas faixas paralelas no sentido Leste-Oeste (propiciando menor autosombreamento da cultura), delimitadas por trilhos com bitola de $1,45 \mathrm{~m}$, sendo o comprimento das parcelas de 2,5 $\mathrm{m}$ com intervalos de $0,5 \mathrm{~m}$ entre as mesmas, onde foi semeada a cultura em três linhas espaçadas de $45 \mathrm{~cm}$, sem bordadura. Sobre os trilhos, desloca-se um protótipo acionado por motor elétrico em velocidade uniforme de $1,33 \mathrm{~km} \cdot \mathrm{h}^{-1}$, ao qual permite-se acoplar uma plaina niveladora, sulcadores, roda compactadora e acessórios para irrigação.
Com o auxílio de enxada rotativa, realizou-se o preparo do solo e a incorporação de $250 \mathrm{~kg} \cdot \mathrm{ha}^{-1}$ de fertilizante (4-20-20) no dia 14 de outubro de 2002. Em 16 de outubro de 2002, realizou-se o nivelamento do solo e os sulcos de semeadura nas profundidades de 0,04, 0,06 e 0,08 m. Em seguida, foram colocadas, manualmente, no interior dos sulcos, 20 sementes de amendoim (IAC Tatú ST) por metro, pré-tratadas com fungicida à base de Thiran. O fechamento dos sulcos foi realizado através de rastelos de madeira para que a superfície voltasse à condição original de nivelamento. Utilizando uma roda metálica de $0,10 \mathrm{~m}$ de largura e de superfície lisa, foi realizada a compactação sobre as linhas de semeadura, colocando-se pesos sobre a mesma, resultando em pesos de $0 ; 98,1$; 196,2 e 294,3 N (10; 20 e 30 kgf, respectivamente). Foram realizadas irrigações diárias, mantendo o solo próximo à sua capacidade de campo.

Foram necessárias quatro pulverizações para o controle de trípes e lagartas, à base de Methamidophos e Deltamethrine, e três pulverizações com fungicida à base de Tiofanato Metílico e Chlorotalonil, para cercosporioses (Mycosphaerella arachidis e M. berkeleyi) e verrugose (Sphaceloma arachidis).

$\mathrm{O}$ número médio de dias para a emergência das plântulas de amendoim foi determinado através de contagens diárias, iniciadas cinco dias após a semeadura, durante 10 dias. Os cálculos foram realizados de acordo com a equação de Edmond \& Drapala (1958).

A altura das plântulas foi avaliada do solo até a inserção da última folha, 15 dias após a semeadura. Aos 110 dias foi efetuada a colheita manual. As vagens foram debulhadas e obteve-se a massa de vagens vazias e grãos.

A produtividade foi obtida através da relação da área colhida por parcela (uma linha de 2,5 m) e a massa de grãos obtida dessa, convertida para a umidade de $8 \%$ e, posteriormente transformada em kg.ha-1.

Os dados foram submetidos à análise de variância e para comparar as médias dos tratamentos utilizou-se o Teste de Tukey, aos níveis de 5\% e 1\% de probabilidade, conforme Gomes (1987).

\section{RESULTADOS E DISCUSSÃO}

\section{Número médio de dias para emergência de plântulas}

Na Tabela 1, verifica-se que não houve interação significativa entre os fatores profundidade de semeadura e compactação do solo sobre a semente, ou seja, os mesmos são independentes. Isso significa que o número médio de dias para emergência de plântulas em função da profundidade de semeadura não é influenciado pela compactação do solo sobre a semente e vice-versa. 
De acordo com a Tabela 1, deduz-se que não houve diferença significativa no número médio de dias para emergência de plântulas para as profundidades de semeadura 0,06 e $0,08 \mathrm{~m}$ por apresentarem valores semelhantes. No entanto, o referido número de dias, para a profundidade $0,04 \mathrm{~m}$, diferenciou-se significativamente das anteriores, obtendo-se o melhor índice de emergência de plântulas, ou seja, menor número de dias. Isso foi observado também por Silva (2002), na cultura do milho e deve-se possivelmente pela menor espessura da camada de solo pelo qual a plântula deve romper para emergir.

Analisando-se o fator compactação do solo sobre a semente, observa-se que não houve diferença significativa desse fator para todas as profundidades de semeadura. Isso significa que não houve influência da compactação do solo sobre a semente no número médio de dias para a emergência de plântulas e que o contato do solo com a semente, necessário para a embebição da mesma para início da germinação, foi satisfatório para todas os pesos impostos.

\section{Altura das plântulas}

Com base na Tabela 1 , verifica-se que não houve interação entre os fatores, ou seja, a compactação do solo sobre a semente e a profundidade de semeadura não dependem uma da outra, indicando que a altura das plântulas não é influenciada simultaneamente pelos dois fatores.

A variável altura das plântulas, em função do fator profundidade de semeadura não evidenciou diferença significativa (Tabela 1), concordando com Justino (1982) que afirma não haver influência da profundidade de semeadura na altura das plântulas, quando estudado o fator isoladamente.

Analisando-se o fator compactação do solo sobre a semente, observa-se que houve diferença significativa entre os pesos $0 \mathrm{~N}$ e 194,2 N, significando que houve influência da compactação do solo sobre a semente para essas cargas. As cargas 98,1 N e 294,3 N foram semelhantes e não diferiram significativamente de $0 \mathrm{~N}$ e 194,2 N, apresentando valores intermediários a esses. Os resultados são contrários aos de Justino (1982), que não observou influência da compactação do solo sobre a semente na altura das plantas de amendoim, quando estudada isoladamente.

\section{Produtividade}

Na Tabela 1, verifica-se que não houve significância na interação pois a produtividade da cultura do amendoim não foi influenciada simultaneamente pelos fatores profundidade de semeadura e compactação do solo sobre a semente.

Tabela 1- Resumo das análises de variância e comparação de médias para as variáveis número médio de dias para emergência, altura das plântulas $(\mathrm{cm})$ e produtividade $\left(\mathrm{kg}_{\text {.ha }} \mathrm{a}^{-1}\right), 2002$, Jaboticabal, SP. ${ }^{(1)}$

\begin{tabular}{cccc}
\hline Fatores & $\begin{array}{c}\mathrm{N}^{\mathrm{o}} \text {. médio de dias } \\
\text { p/ emergência }\end{array}$ & $\begin{array}{c}\text { Altura das plântulas } \\
(\mathrm{cm})\end{array}$ & $\begin{array}{c}\text { Produtividade } \\
\left(\mathrm{kg}^{-1} \mathrm{a}^{-1}\right)\end{array}$ \\
\hline Teste F & $9,75^{* *}$ & $0,26^{\mathrm{NS}}$ & $6,19^{* *}$ \\
$\mathrm{P}$ & $0,18^{\mathrm{NS}}$ & $3,76^{*}$ & $0,22^{\mathrm{NS}}$ \\
$\mathrm{C}$ & $1,04^{\mathrm{NS}}$ & $0,55^{\mathrm{NS}}$ & $0,47^{\mathrm{NS}}$ \\
\hline P x C & 7,70 & 16,24 & 27,12 \\
\hline CV (\%) & & & \\
\hline Profundidade(P) & $7,1 \mathrm{~b}$ & $2,7 \mathrm{a}$ & $6456 \mathrm{a}$ \\
$4 \mathrm{~cm}$ & $8,1 \mathrm{a}$ & $2,9 \mathrm{a}$ & $6752 \mathrm{a}$ \\
$6 \mathrm{~cm}$ & $7,9 \mathrm{a}$ & $2,8 \mathrm{a}$ & $4615 \mathrm{~b}$ \\
$8 \mathrm{~cm}$ & & & $5977 \mathrm{a}$ \\
\hline Compactação $(\mathrm{C})$ & $7,8 \mathrm{a}$ & $2,5 \mathrm{~b}$ & $5965 \mathrm{a}$ \\
$0 \mathrm{~N}$ & $7,7 \mathrm{a}$ & $2,7 \mathrm{ab}$ & $5822 \mathrm{a}$ \\
$98,1 \mathrm{~N}$ & $7,6 \mathrm{a}$ & $3,2 \mathrm{a}$ & $5999 \mathrm{a}$ \\
\hline $194,2 \mathrm{~N}$ & $7,6 \mathrm{a}$ & $2,9 \mathrm{ab}$ & \\
$294,3 \mathrm{~N}$ & & &
\end{tabular}

${ }^{(1)}$ Em cada coluna, para cada fator, médias seguidas de mesmas letras minúsculas não diferem entre si, pelo Teste de Tukey, a 5\% de probabilidade. NS: não significativo $(\mathrm{P}>0,05)$.*: significativo $(\mathrm{P}<0,05)$.**: significativo $(\mathrm{P}<0,01)$. C.V.: coeficiente de variação 
De acordo com essa Tabela, observa-se, também que o fator profundidade de semeadura, apresentou diferença significativa na produtividade da cultura. Para as profundidades $0,04 \mathrm{e} 0,06 \mathrm{~m}$ a produtividade mostrouse semelhante estatisticamente. Na mesma Tabela observase entretanto que, para a profundidade $0,08 \mathrm{~m}$, houve uma diminuição significativa na produtividade em relação às anteriores. Isso mostra que a profundidade de semeadura interfere na produtividade da cultura do amendoim, talvez em razão do maior gasto energético realizado pelas plântulas do amendoim para romper essa camada mais espessa de solo, tornando a planta resultante mais frágil e debilitada, incorrendo em menor produtividade.

Para o fator compactação do solo sobre a semente, constata-se que não houve diferença significativa entre todos os pesos aplicados (0, 98,1, 194,2 e 294,3 N) (Tabela 1), significando que a produtividade da cultura do amendoim independe do peso de compactação do solo sobre a semente, na ocasião do plantio. Esses resultados, entretanto, diferem dos obtidos por Coan et al. (1986) que, estudando a compactação, na cultura do feijão, em profundidade de semeadura constante de $0,075 \mathrm{~m}$, observaram variação na produtividade da mesma. A interação profundidade $\mathrm{x}$ compactação, segundo os autores, não apresentou resultados significativos em seu trabalho, resultado esse também observado no presente trabalho.

\section{Massa seca da parte aérea}

Verifica-se que não houve interação significativa dos fatores profundidade de semeadura e compactação do solo sobre a semente, em relação à massa seca da parte aérea (Tabela 2). $\mathrm{O}$ fator profundidade de semeadura, também não apresentou diferença significativa na produção de massa de matéria seca da parte aérea para as procfundidades 0,04 e $0,06 \mathrm{~m}$. Na mesma tabela observa-se que, para a profundidade $0,08 \mathrm{~m}$, houve uma redução na produção de matéria seca da parte aérea, apresentando diferença significativa em relação às anteriores. Dessa forma, verifica-se que a profundidade de semeadura da cultura do amendoim interfere na produção de massa de matéria seca da mesma, tendo como possível explicação o maior gasto energético, como descrito na variável anterior.

Para o fator compactação do solo sobre a semente, observa-se que não houve diferença significativa entre os pesos aplicados (Tabela 2). Isso mostra que a produção de matéria seca da parte aérea da cultura do amendoim não é

Tabela 2 - Resumo das análises de variância e comparação de médias para as variáveis massa seca da parte aérea $\left(\mathrm{kg} \cdot \mathrm{ha}^{-1}\right)$, massa de vagem vazia $\left(\mathrm{kg} \cdot \mathrm{ha}^{-1}\right)$ e massa de grãos $\left(\mathrm{kg} \cdot \mathrm{ha}^{-1}\right), 2002$, Jaboticabal, SP. ${ }^{(1)}$

\begin{tabular}{|c|c|c|c|}
\hline Fatores & $\begin{array}{l}\text { Massa seca da parte } \\
\text { aérea }\left(\mathrm{kg} \cdot \mathrm{ha}^{-1}\right)\end{array}$ & $\begin{array}{l}\text { Massa de vagem } \\
\text { vazia }\left(\mathrm{kg}^{-} \mathrm{ha}^{-1}\right)\end{array}$ & $\begin{array}{c}\text { Massa de } \\
\text { grãos }\left(\mathrm{kg}^{-h^{-1}}\right)^{-1}\end{array}$ \\
\hline \multicolumn{4}{|l|}{ Teste F } \\
\hline $\mathrm{P}$ & $22,13 * *$ & $4,33^{*}$ & $6,72 * *$ \\
\hline $\mathrm{C}$ & $0,76^{\mathrm{NS}}$ & $0,19^{\mathrm{NS}}$ & $0,06^{\mathrm{NS}}$ \\
\hline $\mathrm{P} \times \mathrm{C}$ & $0,64^{\mathrm{NS}}$ & $0,31^{\mathrm{NS}}$ & $0,54^{\mathrm{NS}}$ \\
\hline $\mathrm{CV}(\%)$ & 15,58 & 29,18 & 27,09 \\
\hline \multicolumn{4}{|c|}{ Profundidade $(\mathrm{P})$} \\
\hline $4 \mathrm{~cm}$ & $1200 \mathrm{a}$ & $1897 \mathrm{a}$ & $4543 \mathrm{a}$ \\
\hline $6 \mathrm{~cm}$ & $1260 \mathrm{a}$ & $1903 \mathrm{a}$ & $4850 \mathrm{a}$ \\
\hline $8 \mathrm{~cm}$ & $830 \mathrm{~b}$ & $1376 \mathrm{~b}$ & $3241 \mathrm{~b}$ \\
\hline \multicolumn{4}{|c|}{ Compactação (C) } \\
\hline $0 \mathrm{~N}$ & $1160 \mathrm{a}$ & $1641 \mathrm{a}$ & $4336 \mathrm{a}$ \\
\hline $98,1 \mathrm{~N}$ & $1110 \mathrm{a}$ & $1588 \mathrm{a}$ & $4200 \mathrm{a}$ \\
\hline $194,2 \mathrm{~N}$ & $1070 \mathrm{a}$ & $1775 \mathrm{a}$ & $4100 \mathrm{a}$ \\
\hline $294,3 \mathrm{~N}$ & $1040 \mathrm{a}$ & $1797 \mathrm{a}$ & $4209 a$ \\
\hline
\end{tabular}

(1) Em cada coluna, para cada fator, médias seguidas de mesmas letras minúsculas não diferem entre si, pelo Teste de Tukey, a 5\% de probabilidade. NS: não significativo $(\mathrm{P} \geq 05)$. *: significativo $(\mathrm{P} \leq 0,05)$. **: significativo $(\mathrm{P} \leq 0,01)$. C.V.: coeficiente de variação 
afetada pelo peso de compactação do solo sobre a semente no momento do plantio.

\section{Massa de vagem vazia}

Para a variável massa de vagem vazia, verifica-se que não houve interação significativa entre os fatores profundidade de semeadura e compactação do solo sobre a semente (Tabela 2), mostrando que essa não é influenciada simultaneamente pelos dois fatores.

De acordo com essa Tabela, observa-se ainda que não houve diferença significativa para a massa de vagem vazia nas profundidades de semeadura 0,04 e 0,06 m. No entanto, a referida massa, para a profundidade $0,08 \mathrm{~m}$, diferenciou significativamente das anteriores, obtendo-se o menor valor por hectare, também tendo como possível explicação para o fato o maior gasto energético para rompimento da camada mais espessa.

Analisando-se o fator compactação do solo sobre a semente, observa-se que não houve diferença significativa desse para a variável massa de vagem vazia.

\section{Massa de grãos}

Com base na Tabela 2, verifica-se que não houve interação significativa, indicando que os fatores profundidade de semeadura e compactação do solo sobre a semente não dependem um do outro, assim a massa de grãos não é influenciada simultaneamente pelos dois fatores.

A variável massa de grãos, em função do fator profundidade de semeadura evidenciou interação significativa (Tabela 2), indicando semelhança entre as profundidades de semeadura 0,04 e $0,06 \mathrm{~m}$. A massa de grãos para a profundidade $0,08 \mathrm{~m}$ mostrou-se inferior às demais, com explicação idem às anteriores.

Analisando-se o fator compactação do solo sobre a semente, observa-se que não houve interação significativa entre os pesos, portanto não houve influência da compactação do solo sobre a semente, na massa de grãos de amendoim.

\section{CONCLUSÕES}

Considerando os resultados obtidos e as condições do ensaio, conclui-se que:

Diferentes compactações do solo sobre a semente, na linha de semeadura não interferem na produtividade da cultura do amendoim.

A profundidade de semeadura de quatro centímetros apresentou o menor número médio de dias para emergência da cultura do amendoim.
A profundidade semeadura de oito centímetros foi a mais desfavorável à cultura do amendoim em termos de produtividade, massa seca da parte aérea, massa de vagem vazia e massa de grãos.

A interação profundidade $\mathrm{x}$ compactação não apresentou correlação, mostrando que os fatores agiram independentemente.

\section{REFERÊNCIAS BIBLIOGRÁFICAS}

ANDRIOLI, I.; CENTURION, J. F. Levantamento detalhado dos solos da Faculdade de Ciências Agrárias e Veterinárias de Jaboticabal. In: CONGRESSO BRASILEIRO DE CIÊNCIA DO SOLO, 27., 1999, Brasília, DF. Anais... Brasília, DF: Sociedade Brasileira de Ciência do Solo, 1999. CDROM.

COAN, O.; ORTOLANI, A. F.; PELLICER, C. A. P. Influência da profundidade de semeadura e compactação do solo sobre a semente, na emergência, desenvolvimento inicial e produtividade do feijoeiro (Phaseolus vulgaris L.). In: CONGRESSO BRASILEIRO DE ENGENHARIA AGRÍCOLA, 15., 1986, São Paulo. Anais... Botucatu: Sociedade Brasileira de Engenharia Agrícola, 1986. p. 76-83.

EDMOND, J. B.; DRAPALA, W. L. The effects of temperature, sand and soil acetone on germination of okra seed. Proceedings America Society Horticultural Science, Alexandria, v. 71, p. 428-434, 1958.

FURLANI, C. E. A.; LOPES, A.; REZENDE, L. C.; SILVA, S. S. S.; LEITE, M. A. Influência da compactaçãodo solo na emergência das plântulas de milho a diferentes profundidades de seneadura. Engenharia na Agricultura, Viçosa, v. 9, n. 3, p. 147-153, 2001.

GOMES, F. P. A estatística moderna na agropecuária. Piracicaba: Associação Brasileira para Pesquisa da Potassa e do Fosfato, 1987. 162 p.

HUMMEL, J. W.; GRAY, L. E.; NAVE, W. R. Soybean emergence from field seedbed environments. Trans. ASAE, Saint Joseph, v. 24, n. 4, p. 872-878, 1981.

JUSTINO, A. Influência da profundidade de semeadura e compactação sobre o solo, na emergência e desenvolvimento inicial do amendoim (Arachis hypogaea L.). 1982. $80 \mathrm{f}$. Monografia (Graduação em Agronomia) - Faculdade de Ciências Agrárias e Veterinárias, Universidade Estadual Paulista, Jaboticabal, 1982. 
ORTOLANI, A. F.; BANZATO, D. A.; BORTOLI, N. M. Influência da profundidade de semeadura e da compactação do solo na emergência e desenvolvimento do sorgo granífero. In: CONGRESSO BRASILEIRO DE ENGENHARIA AGRÍCOLA, 20., 1986, São Paulo. Anais... Botucatu: Sociedade Brasileira de Engenharia Agrícola, 1986. p. 21.

PACHECO, E. P. Avaliação de uma semeadoraadubadora de precisão com modificações no tubo condutor de sementes. 1994. 61 f. Dissertação (Mestrado em Mecanização Agrícola) - Universidade Federal de Viçosa, Viçosa, 1994.

SAVI FILHO, A. Técnicas adequadas para o cultivo do amendoim. [S.1.]: Correio Agrícola, 1980. p. 258-265.

SILVA, R. P. Efeito de rodas compactadoras submetidas a cargas verticais em profundidades de semeadura nas características agronômicas do Milho (Zea mays L.). 2002. 101 f. Tese (Doutorado em Produção Vegetal) Faculdade de Ciências Agrárias e Veterinárias, Universidade Estadual Paulista, Jaboticabal, 2002. 